\title{
Corporéité et créativité, entre traditions et innovations
}

\section{John Didier}

Les disciplines artisanales sont traditionnellement associées à la transmission de gestes techniques dans un contexte scolaire. Devenues les ambassadrices de la créativité dans le cadre de la scolarité obligatoire, ces disciplines techniques aux représentations manuelles nous amènent à repenser les gestes techniques et les savoirs à enseigner aux élèves. En introduisant des démarches de conception au sein de l'agir enseignant en activités créatrices et manuelles, nous réactivons la question de la corporéité, de ces savoirs et de ces techniques corporelles, en phase avec les pratiques sociales d'aujourd'hui. Aussi, développer la créativité en tant capacité à produire des idées innovantes et adaptées, renvoie à enseigner des tâches complexes telles que la résolution de problèmes (Bonnardel, 2006). De ce fait, l'élève apprend à anticiper, analyser et évaluer quels types de ressources devront être mobilisées dans l'action. À mi-chemin entre tradition et innovation, la corporéité nous rend attentifs à ces savoirs liés aux représentations et à l'évolution de ces techniques corporelles.

\section{Introduction}

Nous concentrons notre réflexion sur la corporéité et créativité en nous focalisant sur la discipline des activités créatrices et manuelles enseignées en Suisse romande. Les activités créatrices et manuelles sont souvent réduites à un enseignement orienté sur la transmission de techniques par imitation et par reproduction. Notre approche en didactique se dissocie de cet enseignement. De ce fait, nous introduisons dans la scolarité obligatoire l'enseignement de démarches de conception en vue de développer la créativité, l'autonomie et les compétences chez l'élève (Didier \& Leuba, 2011; Leuba, 2014; Didier, 2012, 2014, 2015). Nous questionnons l'articulation entre corporéité et créativité dans le but d'évaluer l'impact du modèle didactique «conception-réalisation-socialisation» sur la formation d'enseignants généralistes en vue de développer la créativité chez l'élève dans l'enseignement des activités créatrices et manuelles. Pour mieux préciser cette notion de corporéité, nous revenons sur le classement 
des techniques du corps en rapport au rendement humain développé par Mauss (1950/2013). Ce rapport entre transmissions des techniques du corps et rendement humain s'observe de manière apparente dans l'enseignement des activités créatrices et manuelles basées sur la transmission et l'acquisition de gestes techniques orientés sur la production d'objets matériels. Cette discipline se caractérise par un enseignement essentiellement focalisé sur des apprentissages transmis par l'acquisition de techniques corporelles par imitation de gestes et de marches à suivre. Le rapport au corps et au geste technique reste encore profondément ancré dans les traditions et les représentations de cette discipline où la référence à l'activité manuelle est prépondérante. Le deuxième terme spécifiant cette discipline renvoie quant à lui, à la créativité, concept fréquemment défini d'un point de vue psychologique en tant que production nouvelle et adaptée au contexte (Lubart, 2003). Cette rencontre entre l'activité manuelle, lieu priviliégé de la corporéité, et la créativité, cette capacité transversale, apparaît difficile et souvent peu mise en œuvre dans l'enseignement des disciplines techniques (Didier \& Leuba, 2011). Aussi, pour permettre de développer la créativité des élèves, nous introduisons les activités de conception créative dans la formation des enseignants. Les activités de conception créatives requièrent des connaissances techniques, une expertise dans le domaine et de la créativité (Bonnardel, 2006). Dans cette perspective, nous proposons une alternative à l'enseignement par imitation de gestes techniques en introduisant l'activité de conception dévoluée à l'élève (Didier \& Leuba, 2011; Didier, 2014, 2015; Leuba, 2014) comme changement de paradigme disciplinaire. De ce fait, comment enseigner des démarches de conception dans le but de développer la créativité chez l'élève lors de la production d'objets matériels?

\section{Entre productions et apprentissages, du geste physique au geste intellectuel}

Pour mieux comprendre le rôle de la corporéité dans l'enseignement des disciplines techniques, il semble nécessaire de questionner l'épistémologie des activités créatrices et manuelles. La discipline activité créatrice et manuelle en Suisse romande regroupe les travaux manuels, les activités créatrices sur textiles et les activités créatrices. Depuis 2010, ces disciplines techniques sont rattachées au domaine des arts dans le plan d'étude romand. Ces disciplines se caractérisent par des traditions disciplinaires liées à la transmission de techniques et de différents savoirs faisant référence à des gestes et à des pratiques sociales en relation avec une culture des métiers, avec une culture technique (Didier, 2012, 2015). Dès lors, le rôle de la corporéité semble naturellement se poser et renforcer des représentations disciplinaires liées à la répétition de gestes corporels qui donneraient lieu à des savoirs. Paradoxalement, ces apprentissages développés par imitation et transitant par la répétition corporelle de gestes précis et rigoureux ne 
font intervenir que très peu d'habiletés cognitives liées à la synthèse, à l'analyse et à la résolution de problèmes. Ces habiletés cognitives sont nécessaires à l'utilisation de la pensée divergente et de la pensée convergente chez l'élève, dans le but de générer des idées innovantes et adaptées, en d'autres termes, développer sa créativité (Lubart, 2003). L'apprentissage par reproduction de gestes mécanisés renvoie à une forme d'acquisition de techniques et de savoirs qui n'est pas sans rappeler le classement des techniques corporelles explicitées par Mauss (1950/2013):

Les techniques du corps peuvent se classer par rapport au rendement, par rapport aux résultats de dressage. Le dressage, comme le montage d'une machine, est la recherche, l'acquisition de rendement. Ici c'est un rendement humain. Ces techniques sont donc les normes humaines du dressage humain. Ces procédés que nous appliquons aux animaux, les hommes se les sont volontairement appliqués à eux-mêmes et à leurs enfants (Mauss, 1950/2013, p. 374).

Selon Mauss (1950/2013) les techniques corporelles renvoient à une construction du savoir qui n'est pas sans rappeler le dressage. Dans le contexte de la scolarité obligatoire, l'enseignant montre des gestes techniques qui sont ensuite imités par l'élève en vue de produire un objet technique. Ainsi, dans un contexte d'enseignement et de construction de l'humain, se pose cette question des apprentissages de l'élève et de son rapport à l'utilisation du corps comme vecteur de construction du savoir. De ce fait, pour passer du geste répété, de l'action automatisée à un geste intellectuel conscientisé, nous proposons un éclairage théorique fondé sur une approche issue de la didactique professionnelle (Pastré, 2006). En effet, dans tout geste professionnel impliquant le corps, le registre pragmatique apparaît souvent prépondérant. Le moment de réflexion qui suit l'action, de prise de conscience de ce même geste entraîne un changement de registre qui induit une conscientisation du savoir (le registre épistémique) (Pastré, 2006). Ces gestes intellectuels peuvent être développés à l'aide des activités de conception créatives qui impliquent un niveau d'abstraction, d'analyse des gestes techniques à anticiper et de prises de décisions argumentées (Didier \& Bonnardel, 2015).

L'association entre la technique, le rendement et la production, proposée par Mauss (1950/2013) associe la corporéité à une construction du savoir par imitation. Il en va de même avec le sujet agissant sur la matière de manière automatisée, voire mécanisée. L'introduction de la conception comme activité centrale induit des gestes intellectuels spécifiques qui renvoient à une corporéité porteuse d'habiletés cognitives mobilisée par le sujet. Associer la corporéité à la créativité à l'aide d'activités de conception créatives nous amène donc à dépasser ce constat du corps apprenant par des gestes répétés et automatisés pour introduire de l'innovation dans cette relation au savoir. Dans ce rapport au geste innovant, la créativité donne lieu à une nouvelle mimésis où la cognition surpasse le geste automatisé et mécanisé. 
Nous entendons par créativité une "capacité à produire une idée exprimable sous une forme observable ou à réaliser une production qui soit à la fois novatrice et inattendue, adaptée à la situation et (dans certains cas) considérée comme ayant une certaine utilité ou de valeur» (Bonnarel, 2006, p. 95). La créativité soulève donc de nouveaux savoirs qui font appel aux facteurs cognitifs, conatifs, motivationnels et environnementaux (Lubart, 2003). Paradoxalement, pour développer la créativité, il convient de conserver des spécificités disciplinaires liées à la maîtrise de gestes techniques nécessaires à la maîtrise du champ (Lubart, 2003). Ces facteurs sont nécessaires à la concrétisation d'idées nouvelles et adaptées au moment de la production d'un objet matériel. L'enseignement de la créativité nécessite une articulation entre tradition des savoir-faire et innovation dans l'enseignement de nouveaux savoirs.

\section{Introduction du cadre théorique "conception- réalisation-socialisation" dans un contexte de production d'objets matériels et de construction de l'humain}

Pour dépasser ce consensus entre maîtrise de gestes techniques traditionnels et production d'idées innovantes et adaptées, nous introduisons dans l'enseignement des activités créatrices et manuelles des phases de questionnements et d'analyses sur les savoirs et les techniques nécessaires à la conception d'un objet ou d'un projet d'objet.

Figure 1: Image du modèle théorique "conception-réalisation-socialisation»

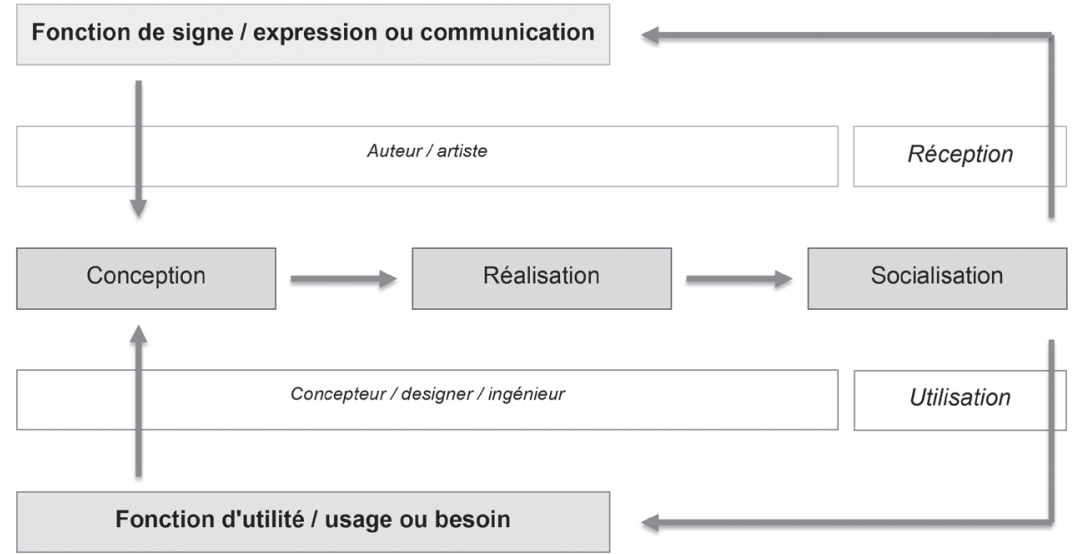


Pour enseigner une créativité contextualisée et maîtrisée (Didier \& Leuba, 2011), nous avons développé un modèle théorique "conception-réalisation-socialisation» (Didier \& Leuba, 2011; Didier, 2014, 2015). Celui-ci introduit des activités de conception dévoluées aux élèves par l'enseignant lors de la fabrication d'un objet matériel. Ce modèle théorique développe une créativité appliquée chez l'élève en permettant de construire des moments d'enseignement qui travaillent différents types d'habiletés cognitives tels que l'analyse, la flexibilité, la synthèse, l'abstraction et la construction d'hypothèses. Ce modèle théorique permet de guider le processus créatif de l'élève lors de la conception et de la réalisation d'un objet. Dans le cadre de la formation des enseignants, la mise en place de ce modèle porte un intérêt significatif aux situations problèmes (Orange, 2005) déléguées aux élèves et plus spécifiquement aux situations problèmes de conception (Bonnardel, 2006). Les situations problèmes se définissent en tant que tâches complexes (Bonnardel, 2006). Ces tâches ne se limitent pas à des actions physiques sur la matière mais englobent des gestes intellectuels intégrant l'analyse, l'émergence d'idées adaptées au contexte ainsi que leur évaluation, la prise de décision, de l'anticipation et la construction d'hypothèses (Didier \& Bonnardel, 2015). La caractéristique des observations retenues se concentre sur l'introduction de la conception au sein de l'enseignement. Ces observations mettent en évidence la construction des objectifs d'apprentissages axés sur la mise en application du modèle "conception-réalisation-socialisation» en tant qu'objet d'apprentissage. Pour approfondir la question des apprentissages, nous travaillons sur l'explicitation des contenus d'enseignements enseignés dans la séquence. Ces savoirs en jeu sont précisés à l'aide des habiletés cognitives issues de la taxonomie d'Anderson et al. (2001).

Pour l'élève, résoudre une situation problème posée par l'enseignant nécessite la mobilisation d'habiletés cognitives telles que: se souvenir, comprendre, appliquer, analyser, évaluer ou créer des solutions innovantes et adaptées tout en tenant compte d'un champ de contraintes.

Les situations problèmes nécessitent de mettre en avant l'autonomisation du sujet en s'appuyant sur la dévolution (Dumas, 2005). Celles-ci font appel à l'esprit scientifique et confrontent le sujet à des problèmes qui ne présentent pas une solution unique dont certains composants sont incertains (Orange, 2005). Le problème de conception qualifié de problème ouvert admet plusieurs solutions de solutions (Fustier, 1989). Ainsi, l'exploration des mécanismes des problèmes de conception renforce la question des apprentissages et l'accès à la cognition du sujet amené à résoudre des problèmes lors de ses actions sur la matière.

La conception fait intervenir une créativité appliquée et rationalisée, où les activités de conception sont considérées comme des activités de résolution de problèmes complexes (Bonnardel, 2006). La conception fait apparaître de nouveaux gestes techniques tels que communiquer ses idées en utilisant différents types de langages (Duboux, 2009). Elle nécessite également d'anticiper et de faire des choix pour concevoir et réaliser un objet. Notre étude propose de 
mettre en évidence les relations entre corporéités et savoirs liés à l'enseignement de gestes techniques issus de pratiques sociales en lien avec les démarches de conception dans la scolarité obligatoire. De ce fait, notre étude apporte un nouveau regard didactique lié à la corporéité des savoirs basés sur les démarches de conception, liant ainsi innovation et tradition dans l'enseignement.

\section{Méthodologie}

\section{La caractéristique des observations retenues}

Nous employons dans notre recherche une approche clinique et expérimentale focalisée sur les transactions des acteurs à propos des objets d'enseignement/ apprentissage (Leutenegger, 2009). Cette recherche s'inscrit dans le cadre de la formation de futurs enseignants généralistes du cycle un et deux à la Haute Ecole pédagogique [HEP Vaud]. Nous focalisons notre approche sur l'analyse didactique permettant d'identifier et de spécifier les différents savoirs mobilisés qui apparaissent dans une démarche de conception d'objets. Les participants de cette recherche sont composés de 45 étudiantes et étudiants ${ }^{1}$ en troisième année de formation d'enseignants généralistes destinés à enseigner au cycle deux et de 90 étudiants en deuxième année de formation d'enseignants généralistes destinés à enseigner au cycle un. Le temps d'implémentation du modèle théorique "conception-réalisation-socialisation" dans le cadre de leur pratique enseignante s'est échelonnée sur une période d'un semestre voir d'une année en fonction du type de stages. Les données ont été récoltées sur deux années de formation durant lesquelles nous avons récolté les éléments suivants: dossier d'analyse de la pratique enseignante des étudiants en formations; des moments filmés durant les stages qui mettent en évidence des moments clefs où les élèves ont été mis en posture de concepteur amenés à résoudre des tâches complexes; des planifications semestrielles focalisées sur le modèle "conception-réalisation-socialisation» en tant qu'objet d'apprentissage.

Les différentes traces vidéo et d'analyse de pratiques issues des interactions entre les enseignants en formation et les élèves, se concentrent sur l'activité de conception travaillée à l'aide de situations problèmes dévoluées à l'élève.

\section{Les critères de sélection}

Les critères de sélection de cette étude sont déterminés par: a) le cycle des élèves dans lequel interviennent les enseignants en formation en lien avec leur stage en classe (nous nous sommes concentrés sur le Cycle 1, enfants âgés de 4 à 8 ans, et le Cycle 2, enfants âgés de 9 à 12 ans), b) le choix disciplinaire orienté sur les activités créatrices et manuelles, c) l'enseignement d'un moment d'activité créatrice centrée sur l'activité de conception, d) la mise en œuvre explicite d'une posture de concepteur chez l'élève (l'élève doit être amené à résoudre un problème présentant plusieurs solutions et donc anticiper et choisir des 
solutions en fonction de contraintes données), e) des moments d'enseignement dans lesquels l'enseignant pose des questions sur la production de l'élève afin d'accéder à la réflexion de celui-ci.

\section{L'instrumentation}

Pour répondre à notre question de recherche (comment enseigner des démarches de conception dans le but de développer la créativité chez l'élève lors de la production d'objets matériels), nous procédons par un recensement des gestes professionnels (Bucheton \& Soulé, 2009) mis en œuvre pour introduire des actes de dévolution de l'activité de conception de l'élève mis en posture d'apprenti concepteur. Nous rattachons notre approche empirique à une conception sémiotique des apprentissages:

Adhérer à une telle conception revient pour nous à admettre qu'apprendre implique non seulement un engagement dans l'activité et une mobilisation des ressources collectives, mais présuppose par ailleurs une participation à des espaces de communication et plus généralement un accès à des significations véhiculées par le langage et d'autres systèmes de signes (des gestes, des postures, des actions, des objets, des schémas, des images, etc.) (Filliettaz, Saint-Georges \& Due, 2008).

Notre dispositif facilite la saisie des données focalisées sur les codes de communication qui interviennent aussi bien au niveau de l'activité enseignante, de l'activité de l'élève, mais également au niveau de systèmes de signes (schémas, croquis, objets, gestes, postures) (Filliettaz, Saint-Georges \& Due, 2008). Ces différentes traces de conception permettront de focaliser les questionnements des étudiants sur la mobilisation des savoirs par les élèves afin de mesurer le degré d'appropriation de la situation de conception.

\section{Le déroulement}

Le déroulement de cette recherche empirique s'effectuera en deux temps à partir des analyses issues de moments d'enseignement des futurs enseignants généralistes en formation. Dans le cadre de deux modules de formation en activités créatrices, les étudiants mettent en œuvre des situations d'enseignement/apprentissage en se focalisant sur la mise en œuvre d'une posture de concepteur chez l'élève. Par cette orientation didactique, ils identifient les contenus d'enseignements spécifiques à l'activité de conception d'un objet en accédant aux savoirs mobilisés par l'élève. L'analyse de gestes enseignants centrés sur la transposition didactique des démarches de conception donne lieu à un travail d'analyse qui est observé dans un premier temps, de manière collective en séminaire, puis, dans un second temps, par l'équipe de didacticiens.

Une double démarche de transposition didactique est donc organisée. Temps 1: tout d'abord, les étudiants se focalisent sur leur planification afin de répondre aux exigences du formateur et à ceux des praticiens formateurs. Temps 2: 
l'étudiant conçoit des situations d'enseignement/apprentissage en privilégiant des situations problèmes dévoluées aux élèves. L'introduction et l'exploitation d'objets intermédiaires (cahier des charges, croquis, maquettes) permettent de relever et d'observer les stratégies de résolutions du problème.

La mise en ouvre des conceptions didactiques au sein de la formation des étudiants en formation se base sur la construction d'un vocabulaire permettant d'identifier et de mesurer les gestes enseignants appropriés pour développer l'activité de conception chez l'élève.

La construction de ce vocabulaire didactique permet aux étudiants de s'approprier les mécanismes de la transposition didactique. De plus, ceci facilite la construction d'outils d'analyses de l'activité enseignante, mais également de l'activité de l'élève. Notre intérêt consiste donc à renforcer l'articulation entre pratique professionnelle et identification des contenus d'enseignement.

\section{La méthode d'analyse des données}

La méthode d'analyse des données se fonde sur une analyse des tâches observées (Leutenegger, 2009) des vidéos des étudiants dans le but d'identifier et de relever les gestes professionnels centrés sur les activités de conception dévoluées aux élèves. Ces vidéos sont analysées afin d'accéder à un second degré de niveau d'information qui permet de dresser un synopsis de leçons (Dolz, Rouveaux \& Schnnewly, 2006). Le corpus de ces vidéos donne lieu à une mise en évidence des spécificités et des générécités dans la pratique professionnelle associée à la dévolution de l'activité de conception dans l'enseignement des descriptions techniques. Notre analyse relève donc les orientations didactiques ainsi que la problématique des contenus didactiques.

À travers les extraits de retranscription, nous mettons en évidence les objets d'enseignement tels qu'ils apparaissent dans le milieu didactique évoluant dynamiquement (indices mésogénétiques) par les contenus de type déclaratif, par exemple, des notions ou du lexique indiqué avec souligné (indice) et les savoir-faire à l'aide d'un double indice (indice) (Rickenmann, 2014). Le second type d'indices met en évidence les postures ou les dispositions à l'action (les indices topogénétiques) indiquées avec des pointillés simples (indịceş) et ceux concernant les pratiques locales à la situation didactique indiquée par soulignés en pointillés (indices).

Notre premier exemple fait référence à une activité didactique proposée à des élèves de $4^{\mathrm{e}}$ année HarmoS (enfants âgés de 7-8 ans) dans une école primaire du canton de Vaud par une stagiaire en stage à temps partiel sur une année. L'activité en question dure 18 minutes et comporte deux éléments clefs: 1) une analyse collective de l'objet matériel à produire qui donne lieu à l'introduction du cahier des charges $(8 \mathrm{~min}$.$) , 2) la réalisation individuelle d'une marche à$ suivre sous forme de croquis (11 min.), 3) l'accès aux stratégies de réflexion d'une élève. 


\section{Exemple 1}

Première partie du second moment d'enseignement: explicitation de la consigne

Première tâche: «Les étapes de la marche à suivre de l'arbre à perles » $(\min 0: 00)$

ta 1. Ens. Maintenant qu'on a rempli le début de notre cahier des charges, je. voudrạis que vous alliez faire un croquis ...

ta 2. El1. Un croquis

ta 3. Ens. De quelles étapes, par quelles étapes je vais devoir passer pour faire le bricolage. Un croquis c'est 1 dessin.

ta 4. Els. (groupe). Ahh

ta 5. Ens. L'aimerais que vous fassiez, que vous dessiniez les étapes pour faire le bricolage. Dans quel ordre est-ce que je vais faire ce bricolage?

Vous allez essayer chacun de votre côté de faire cela et puis après on regardera tous ensemble. D'accord?

ta 6. El3. On doit faire quoi? On doit faire un dessin, mais de quoi?

ta 7. Ens. Ie veux que vous dessiniez les différentes étapes pour faire le bricolage. Par exemple en 1 ie dois aller chercher un caillou. En 2 ie dois aller chercher des perles. L'aimerais que vous réfléchissiez dans quel ordre vous allez faire votre bricolage et que vous me dessiniez ces différentes étapes. Est-ce que vous avez des questions?

ta 8. El 1. Euh non?

ta 9. Ens. Paul ${ }^{2}$

ta 10. El 2. Ję ne comprends pas comment on pourrait le dessiner, ie trouve qu'on devrait plus l'écrire?

ta 11. Ens. Alors si tu penses que c'est plus facile de l'écrire, soit faire un dessin où l'écrire, ou les deux

$(\min 1: 20)$

Dans cette leçon, l'enseignante définit la situation problème qu'elle délègue aux élèves en leur demandant de dessiner la marche à suivre pour réaliser l'objet matériel. Cet objet, l'arbre à perles, a été analysé collectivement en début de leçon. Le fait de débuter ce moment d'enseignement par une analyse fonctionnelle de l'objet permet aux élèves de nommer et d'identifier les matériaux nécessaires à la réalisation de l'artefact. La situation problème a pour objectif de renforcer l'autonomie de l'élève et sa capacité à anticiper. Nous quittons un enseignement transmissif pour privilégier un enseignement par problèmes qui habitue l'élève à réfléchir et à communiquer graphiquement une marche à suivre de l'objet à réaliser. Nous noterons dans le tour d'action 1 (le terme ta renvoie au tour d'action) que la focalisation sur la consigne induit une redéfinition de celle-ci par l'enseignante - qui ajoute des précisions sur les termes clefs. L'enseignante quitte progressivement une consigne ouverte et floue pour préciser les termes et 
les redéfinir (ta 3 «un croquis c'est un dessin»). Ceci permet de contextualiser un savoir dans une situation où celui-ci devient un enjeu clef de l'activité. L'activité de conception induit donc, de la part des élèves, une capacité à s'approprier un problème, ici une marche à suivre, pour synthétiser, par un système de représentations graphiques, les étapes clefs et les matériaux nécessaires à la réalisation de l'objet.

Dans le contexte de cette recherche, nous introduisons auprès des enseignantes en formation le cahier des charges. Cet outil induit un pilotage de l'activité de conception en introduisant une progression au niveau de l'autonomie et de l'anticipation de l'élève en fonction des tâches prescrites. Le cahier des charges a pour fonction de comprendre les différentes contraintes liées à la conception, à la réalisation et à la socialisation (utilisation/réception) de l'objet matériel. Le cahier des charges introduit une vision d'ensemble de la réalisation de l'objet en demandant à l'élève de conscientiser par écrit ou graphiquement la marche à suivre nécessaire à la réalisation de l'objet. Dans ce contexte d'enseignement, il est probable que l'élève ( $\mathrm{El} 2)$ soit déjà familiarisé avec cette manière de procéder.

Nous relevons dans l'analyse de ce moment d'enseignement, la difficulté de l'enseignante à employer le terme «activité de conception». Cette étudiante en formation introduit une activité de conception en revenant essentiellement sur un registre pragmatique en faisant essentiellement référence au savoir-faire (la consigne faire le bricolage est énoncée cing fois). L'introduction de la notion d'étape du bricolage est un élément clef, car elle met en place une conscientisation de la succession des actions à réaliser qui permet de développer l'anticipation chez l'élève (ta 3 De quelles étapes, par quelles étapes je vais devoir passer / ta 5 Dans quel ordre). Toutefois, l'enseignante n'explicite pas clairement les raisons de ses choix didactiques en expliquant le choix de sa consigne au sujet d'une marche à suivre dessinée. Ce même choix donne lieu à un consensus suite à la remarque de l'élève ( $\mathrm{El} 2)$ (ta 11. Ens. Alors si tu penses que c'est plus facile de l'écrire, soit faire un dessin ou l'écrire, ou les deux).

\section{Exemple 2}

Deuxième partie: Accès à la cognition de l'élève. (0:00)

Deuxième tâche: Moment d'analyse.

L'enseignante et l'élève sont devant le croquis de l'élève en question qui comporte 3 étapes pour la réalisation de l'objet matériel (l'arbre à perle).

ta 1. Ens. Explique-moi ce que tu as fait

ta 2. El1. (L'élève montre la première étape de son dessin) D'abord, j’ai dessiné les petites barres de fil de fer

ta 3 Ens. Donc tu as dessiné des morceaux de fil de fer. D'accord

ta 4. El1. Après j’ai mis les perles et puis la pierre, alors 
ta 5. Ens. Donc ça c'est ton matériel (l'enseignante montre du doigt la première étape du dessin) le fil de fer, les perles, et la pierre.

ta 6. El1. Je vais aller chercher le matériel. Ensuite

ta 7 . Ens. Ensuite,

ta 8. El1. (pointe du doigt la deuxième étape du dessin). Il faut rassembler $\underline{\text { le }}$ fil avec les perles.

ta 9. Ens. Donc il faut mettre les perles dans les fils. D'accord

ta 10. El1. Et Puis après, il faut rassembler le caillou avec l'arbre (montre de son doigt la troisième étape de son dessin).

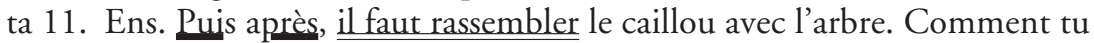
fais pour que les perles tiennent ici? (Pointe avec son doigt)

ta 12. El1. Bein, on les tourne (elle mime le geste avec les deux mains).

ta 13. Ens. Les tourner, d'accord. Et puis comment tu vas faire pour rassembler tous les morceaux de fils pour en faire un arbre? (pointe de son doigt la première et la troisième étape du croquis)

ta 14. El1. 1 fil de fer ... deux, 1 fil de fer qui se trouve sur les côtés puis après qui revient s'emmêler avec les autres (dessine le geste avec son doigt).

ta 15. Ens. Donc on met tous les fils de fer ensemble?

ta 16. El1. oui

ta 17 Ens: Ok. merci.

$(2: 15)$

Le deuxième exemple se concentre sur un moment d'accès à la cognition de l'élève par l'enseignante, fondé sur l'explicitation orale de la marche à suivre dessinée. Nous avons choisi ce moment d'enseignement, car il caractérise un moment remarquable où la question de la corporéité et de la créativité à travers les activités de conception créatives prend un tout autre sens que celui traditionnellement assigné aux activités créatrices. En effet, il ne s'agit pas de répéter un geste en le reproduisant par imitation. Au contraire, l'élève placé en posture d'apprenti concepteur de sa marche à suivre nomme et conscientise les choix et les décisions liées à une projection de la réalisation. La situation problème dévoluée à l'élève, induit une communication graphique à l'aide d'un croquis des différentes étapes, accompagnée du commentaire oral. L'enseignante questionne l'élève sur la cohérence de cette représentation graphique en vue d'évaluer de manière formative sa capacité à anticiper (ta1. Ens. Explique-moi ce que tu as fait). Les gestes de l'élève mîment la technique d'assemblage par insertion, torsion et enroulement des perles avec les fils de fer en vue de représenter l'irrégularité des branches d'un arbre. Ces gestes accompagnent des explicitations qui peinent à trouver une formulation précise en fonction de la technique requise. Dans la ta12 (elle mime le geste de torsion et d'assemblage avec les deux mains), l'élève utilise le geste pour signifier la technique de l'assemblage des perles et du fil de fer. 


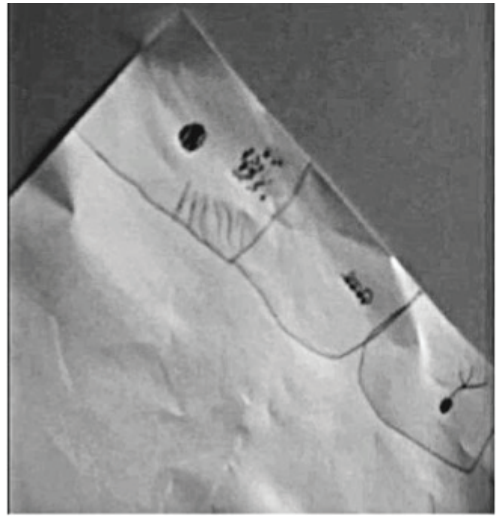

Le croquis intervient comme un artefact cognitif qui permet à l'élève de s'appuyer sur un support graphique à partir duquel il explicite sa marche à suivre.

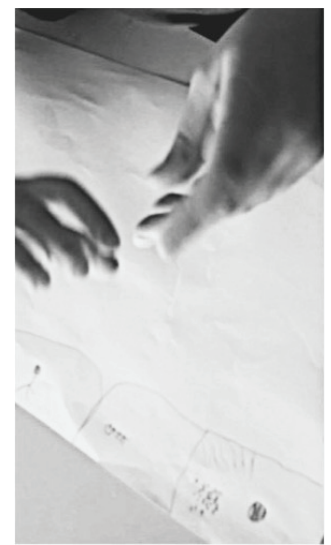

Le geste devient support d'une explicitation orale de techniques (associer différents objets entre eux par enroulement et par torsions) qui amène l'élève à passer du registre pragmatique vers un registre épistémique (Pastré, 2006). Ce moment d'enseignement nous renseigne sur le changement de paradigme disciplinaire où la technique ne se voit plus réduite à un apprentissage par imitation de gestes corporels. L'élève est amené à décider et à expliciter ses choix en vue de conscientiser les savoirs mobilisés qui donnent progressivement lieu à une conceptualisation de l'action (Pastré, 2006). Les apprentissages qui apparaissent dans l'activité constructive (Pastré, 2006) transitent par un changement d'étayage de l'enseignante qui questionne l'élève sur ses propres stratégies d'apprentissages. En répétant les explications de l'élève (ta 3, ta 9, ta 11, ta 13), en proposant des synthèses (ta 5: Donc ça c'est ton matériel) et en la questionnant sur ses choix (ta 11, Comment tu fais pour que les perles tiennent ici? /ta 15. Ens. Donc on met tous les fils de fer ensemble?) l'enseignante modifie son étayage pour favoriser la conscientisation des savoirs mobilisés dans l'action par l'élève. La construction d'une temporalité ponctuée par une série de validations renforce la progression narrative de l'élève. Dans cette séquence, les gestes corporels accompagnent des habiletés cognitives de l'élève tels que décrire, nommer, analyser, évaluer (Anderson et al., 2001). Grâce à ces termes, nous explicitons le changement de paradigme disciplinaire enseigné à l'aide du modèle "conception-réalisation-socialisation» qui donne lieu à l'enseignement de nouveaux savoirs. Ces savoirs ne se limitent plus à imiter des techniques corporelles, mais intègrent cette question de l'innovation dans les apprentissages en amenant l'élève à produire des idées innovantes et adaptées au contexte.

\section{Les considérations éthiques}

Pour revenir sur les considérations éthiques de cette recherche, il semble utile de rappeler la stratégie d'accès à la cognition de l'élève à l'aide des traces graphiques, et/ou écrites à partir desquelles transitent les commentaires des élèves. Cette 
manière de procéder permet une intrusion dans la classe tout en conservant l'anonymat des élèves. De ce fait, sur la vidéo l'enseignante devient l'unique personne reconnaissable et visible au moment de l'énonciation de la consigne. Les traces graphiques interviennent comme des intermédiaires et des médiateurs entre les questions de l'enseignante et les réponses des élèves. Cette recherche se construit donc à partir des traces qui donnent lieu à des savoirs explicités et questionnés. L'enseignante en formation peut donc aisément identifier un moment-clé dans sa séquence d'enseignement apprentissage tout en s'appuyant sur un artefact qui donne lieu à un moment d'étayage orienté sur la métacognition de l'élève.

\section{Résultats obtenus}

Cette recherche fait apparaître une amélioration des gestes professionnels des enseignants en formation. Les étudiants-enseignants en formation ayant participé à ce dispositif de recherche très proche d'une étude de cas ont été amenés à quitter délibérément un enseignement transmissif traditionnellement orienté sur la transmission de savoir-faire. Ils ont pu introduire dans leur enseignement la dévolution de tâches complexes telles que la résolution de problèmes de conception. Ces étudiants ont pu accéder de manière explicite et planifiée à la cognition de leur élève dans le cadre de l'enseignement de démarches de conception. Le passage d'un registre pragmatique des gestes enseignants vers un registre épistémique (par une capacité à d'évoluer l'activité de conception à l'élève) a donné lieu à la construction de gestes professionnels analysés et conceptualisés. La construction de ces gestes professionnels a été travaillée de manière individuelle par l'étudiant sous la forme d'un dossier, mais également de manière collective pendant les séminaires. Cette recherche fait émerger une distanciation opérée par l'étudiant sur sa propre pratique professionnelle et son impact sur la construction des savoirs chez l'élève mis en posture de concepteur.

\section{Conclusion}

Les disciplines artisanales sont traditionnellement associées à la transmission des gestes professionnels dans un contexte scolaire. Devenues à travers leur évolution les ambassadrices de la créativité dans le cadre de la scolarité obligatoire, ces disciplines nous amènent à repenser les gestes techniques et les savoirs à enseigner à l'élève. Notre parti pris concernant l'introduction des démarches de conception au sein de l'agir enseignant en activités créatrices et manuelles remet la question de la corporéité, de ces savoirs et de ces techniques corporelles en phase avec les pratiques sociales d'aujourd'hui. Aussi, développer la créativité en tant capacité à produire des idées innovantes et adaptées, renvoie à enseigner des tâches complexes telles que la résolution de problèmes (Bonnardel, 
2006). De ce fait, l'élève apprend à anticiper, analyser et évaluer quels types de ressources devront être mobilisées dans l'action. À mi-chemin entre tradition et innovation, la corporéité nous rend attentifs à ces savoirs liés à la représentation et à l'évolution de ces techniques corporelles. Nous quittons une compréhension de la technique corporelle rattachée au rendement et à la production (Mauss, 1950/2014). Dans une perspective d'innovation et de construction, ces techniques corporelles renvoient à la construction des savoirs chez l'élève pour l'amener à apprendre à anticiper, à choisir et à communiquer sur les choix et les décisions d'aujourd'hui et de demain.

\section{Notes}

1 Dans le cadre de la formation, ces étudiantes et ces étudiants sont des enseignantes et des enseignants en formation. Nous utiliserons dans cet article la dénomination : enseignantes et enseignants en formation pour nous y référer.

2 Prénom de substitution

\section{Références}

Anderson, L.W (Éd.), Krathwohl, D.E (Éd.), Airasian, P.W., Cruikshank, K.A., Mayer, R.E., Pintrich, P.R., Raths, J. \& Wittrock, M.C. (2001). A taxonomy for learning, teaching, and assessing: A revision of Bloom's Taxonomy of Educational Objectives [Complete edition]. New York: Longman.

Bonnardel, N. (2006). Créativité et conception. Approches cognitives et ergonomiques. Marseille: Solal.

Bonnardel, N. (2009). Activités de conception et créativité: de l'analyse des facteurs cognitifs à l'assistance aux activités de conception créatives. Le Travail Humain, 72(1), 5-22.

Bucheton, B. \& Soulé, Y. (2009). Les gestes professionnels et le jeu des postures de l'enseignant dans la classe: un multi-agenda de préoccupations enchâssées. Éducation et didactique, 3(3), 29-48.

Debord, G. (1992). La société du spectacle. Paris: Gallimard.

Deforge, Y. (1990). L'ouvre et le produit. Seyssel: Champ Vallon.

Deforge, Y. (1993). Le graphisme technique et son histoire et son enseignement. Seyssel: Champ Vallon.

Didier, J. \& Leuba, D. (2011). La conception d'un objet: un acte créatif. Prismes, 15, 32-33.

Didier, J. (2012). Culture technique et éducation. Prismes, 16, 14-15.

Didier, J. (2014). La mise en œuvre de la créativité dans l'enseignement des activités créatrices et techniques. In Ph. Losego (Éd.), Actes du colloque "Sociologie et didactiques: vers une transgression des frontières ", 13 et 14 septembre 2012 (pp. 260-270). Lausanne: Haute Ecole Pédagogique de Vaud. Consulté le 11.12.2014 dans http://www.hepl.ch/sociodidac/actes/

Didier, J. (2015). Concevoir et réaliser à l'école. Culture technique en Suisse romande. In Y. Lequin et P. Lamard (Éd.), Eléments de démocratie technique (pp. 227-238). Sevenans: UTBM.

Didier, J. \& Bonnardel, N. (2015). Activités créatives et innovations pédagogiques dans le domaine du design. In N. Bonnardel, L. Pellegrin \& H. Chaudet (Éd.), Actes du 8ème colloque de Psychologie Ergonomique - EPIQUE 2015 (pp. 165-173). Paris, France: Arpege Science Publishing.

Dolz, J., Ronveaux, Chr. \& Schneuwly, B. (2006). Le synopsis: un outil pour analyser les objets enseignés. In M.J. Perrin-Glorian \& Y. Reuter (Éd.), Les méthodes de recherche en 
didactiques (pp. 175-190) [Éducation et didactiques]. Villeneuve d'Ascq: Presses universitaires du Septentrion.

Duboux, C. (2009). Le dessin comme langage. Lausanne: PPUR.

Dumas, G. (2005). Questions orales à Guy Brousseau dans Salin. In M-H. Clanché et P. Sarrazy (Éd.), Sur la théorie des situations didactiques (pp. 34-38). Grenoble: La Pensée Sauvage.

Filliettaz, L., De Saint-Georges, I. \& Duc, B. (2008). «Vos mains sont intelligentes!» Interactions en formation professionnelle initiale. Genève: Cahier de la section des Sciences de l'Education.

Fustier, M. (1989). La résolution de problème: méthodologie de l'action. Paris: Editions ESF \& Librairies Techniques.

Leutenegger, F. (2009). Le temps d'instruire Approche clinique et expérimentale du didactique oridinaire en mathématique. Berne: Peter Lang.

Mauss, M. (1950/2013). Sociologie et anthropologie (Ouvrage original publié en 1950). Paris: PUF.

Orange, C. (2005). Problème et problématisation. Aster, 40, 3-11.

Leuba, D. (2014). Créatif en AC\&M... oui, mais comment? Revue Educateur, 2. 14, 6-7.

Lubart, T. (2003). Psychologie de la créativité (2e éd.). Paris: Armand Colin.

Pastré, P. (2006). Apprendre à faire. In E. Bourgeois \& G. Chapelle (Éd.), Apprendre et faire apprendre (pp. 109-117). Paris: PUF.

Rickenmann, R. (2014). La construction sociale de l'émotion esthétique: dimensions transactionnelles des rapports à l'objet culturel en classe. In Ch. Moro et N. Muller-Mirza (Éd.), Sémiotique, culture et développement psychologique (pp. 227-294 Villeneuve d'Asq: Presses Universitaires du Septentrion.

Sonntag, M. (2007). La conception au cœur de la formation professionnelle. Les Sciences de l'éducation - Pour l'Ère nouvelle, 40, 59-78.

Mots-clés: Corporéité, créativité, conception, production, apprentissages

\section{Körperlichkeit und Kreativität, zwischen Tradition und Innovation}

\section{Zusammenfassung}

Werkkünstlerische Disziplinen sind traditionell mit der Übertragung von technischen Gesten in einem schulischen Kontext verbunden. Diese technischen Disziplinen, in einem manuellen Ansatz verankert, sind im Rahmen der Schulpflicht Botschafter der Kreativität geworden und führen uns dazu, den Unterricht von manuellen Gesten umzudenken. Durch die Einführung von Design-Ansätzen in den Lehrmethoden, in Form von kreativen und praktischen Aktivitäten, werden die Fragen der Körperlichkeit, des Wissens und der körperlichen Techniken reaktiviert im Einklang mit den gegenwärtigen gesellschaftlichen Bräuchen. Außerdem bezieht sich das Entwickeln der Kreativität als Produktionskapazität für angepasste innovative Ideen auf die Lehre von komplexen Aufgaben wie der Problemlösung.

Schlagworte: Körperlichkeit, Kreativität, Entwurf, Fertigung, das Lernen 


\section{Corporeità e creatività, fra tradizioni e innovazioni}

\section{$\mathrm{Riassunto}$}

Le discipline artigianali sono tradizionalmente associate alla trasmissione dei gesti tecnici nell'ambito scolastisco. Diventate ambasciatrici della creatività nella scuola dell'obbligo, queste discipline tecniche dalle rappresentazioni manuali ci portano a ripensare i gesti tecnici e i saperi da insegnare. Introducendo delle pratiche di concezione nell'ambito della didattica delle attività creatrici e manuali, riattiviamo la questione della corporeità, di questi saperi e di queste tecniche corporali, in fase con le attuali pratiche sociali. Così, sviluppare la creatività in quanto capacità a produrre delle idee innnovanti e adeguate, porta a insegnare dei compiti complessi, come la soluzione di problemi.

Parole chiave: Corporeità, creatività, progettazione, produzione, apprendimento.

\section{Corporeality and creativity, between tradition and innovation}

\section{Summary}

Craft disciplines are traditionally associated with the transmission of technical gestures in a school setting. Since they became, within the context of compulsory education and despite themselves, the ambassadors for creativity, these technical disciplines embedded in manual representations, leads us to rethink the teaching technical gestures and the knowledge to be taught to students. By introducing design approaches within the acting of the creative and manual activities teacher, we reinstate the issue of corporeality, of knowledge and body techniques in line with nowadays social practices. Also, fostering the development of creativity as an ability to produce innovative and tailored ideas refers to teaching complex tasks such as problem solving.

Keywords: Corporeality, creativity, design, production, learning 